See discussions, stats, and author profiles for this publication at: https://www.researchgate.net/publication/350416494

\title{
Some Traits of the Biology and Epigean Behavior of Sisyphus schaefferi (Linnaeus) (Coleoptera: Scarabaeidae: Scarabaeinae: Sisyphini)
}

Article in The Coleopterists Bulletin · March 2021

DOI: 10.1649/0010-065X-75.1.75

CITATIONS

0

3 authors, including:

Enrico Barbero

Università degli Studi di Torino

87 PUBLICATIONS 486 CITATIONS

SEE PROFILE
READS

5

Some of the authors of this publication are also working on these related projects:

Taxonomy and systematics of the Afrotropical dung beetles (Coleoptera, Scarabaeinae) View project 


\section{Some Traits of the Biology and Epigean Behavior of Sisyphus schaefferi (Linnaeus) (Coleoptera: Scarabaeidae: Scarabaeinae: Sisyphini)}

Authors: Rizzotto, Maurizio, Negro, Matteo, and Barbero, Enrico

Source: The Coleopterists Bulletin, 75(1) : 75-85

Published By: The Coleopterists Society

URL: https://doi.org/10.1649/0010-065X-75.1.75

BioOne Complete (complete.BioOne.org) is a full-text database of 200 subscribed and open-access titles in the biological, ecological, and environmental sciences published by nonprofit societies, associations, museums, institutions, and presses.

Your use of this PDF, the BioOne Complete website, and all posted and associated content indicates your acceptance of BioOne's Terms of Use, available at www.bioone.org/terms-of-use.

Usage of BioOne Complete content is strictly limited to personal, educational, and non - commercial use. Commercial inquiries or rights and permissions requests should be directed to the individual publisher as copyright holder.

BioOne sees sustainable scholarly publishing as an inherently collaborative enterprise connecting authors, nonprofit publishers, academic institutions, research libraries, and research funders in the common goal of maximizing access to critical research. 


\title{
Some Traits of the Biology and Epigean Behavior of Sisyphus SchaEFferi (Linnaeus) (Coleoptera: Scarabaeidae: Scarabaeinae: Sisyphini)
}

\author{
Maurizio Rizzotto \\ via Gropello 14 \\ I-10138 Torino, ITALY \\ RZZMRZ@fastwebnet.it \\ Matteo Negro \\ via Martiri della Libertà 42 \\ I-13818 Tollegno (BI), ITALY \\ negro.matteo@gmail.com \\ AND \\ ENRICO BARBERO \\ Dept. of Life Sciences and Systems Biology \\ University of Turin, via Accademia Albertina 13 \\ I-10123 Torino, ITALY \\ enrico.barbero@unito.it
}

\begin{abstract}
A two-year field study on selected traits of the biology of the scarabaeine dung roller Sisyphus schaefferi (Linnaeus) was conducted in a natural park in the western Po Valley in northwestern Italy. Despite laboratory studies carried out over the past few decades, several aspects of the epigean behavior of the species in the wild remain uncertain. Our study revealed that 1) the species is active from early spring to late summer, with reproductive pairs being observed from mid-spring to midsummer; 2) sexual dimorphism in body weight and length is absent; 3) mating individuals are significantly heavier and longer than non-mating individuals; 4) metatibial length increases isometrically with body length and weight; 5) size and weight of the brood balls are isometric with respect to the length and weight of the male of the reproductive pair; 6) brood balls are significantly larger and heavier than food balls, and, whereas brood ball size and weight decrease as air temperature increases, food ball size and weight increase as air humidity rises, but only in non-reproductive periods; 7) pushing and pulling roles are not sex-dependent and can be reversed during the rolling process; 8) combat for balls can involve up to six individuals simultaneously.
\end{abstract}

Keywords: dung beetles, rolling behavior, feeding behavior, reproductive behavior, food ball, brood ball

DOI.org/10.1649/0010-065X-75.1.75

\section{INTRODUCTION}

Roller dung beetles are coprophagous Scarabaeidae that display a peculiar kind of food relocation behavior (Halffter and Edmonds 1982; Zunino 1992). They usually make a ball from the dung pad, then roll it a distance to bury it in a suitable place (with minor exceptions in some South African species of Sisyphus Latreille, see Paschalidis 1974). When this behavior occurs for alimentary purposes, a single individual of either sex acts alone to fashion and roll a food ball. When the goal is nesting, a male or, more rarely, a female makes the ball (brood ball), the partner joins in, and the pair collaborate in rolling and burying the ball. Finally, the female releases an egg in the ball, after remodeling it or not, and leaves the nest (Halffter and Matthews 1966).
As a rule, rolling behavior occurs in four tribes: Scarabaeini, Gymnopleurini, Deltochilini and Sisyphini. Nevertheless, these taxa do not represent a monophyletic clade (Tarasov and Dimitrov 2016; Tarasov and Génier 2015). According to the most recent literature, rolling behavior arose independently several times within the Scarabaeinae (Philips et al. 2004). The traits of the epigean behavior (mainly ball rolling) are not uniform in the four tribes. Among the mainly Old World Sisyphini, the life history of the southern Palearctic species Sisyphus schaefferi (Linnaeus) has been carefully investigated by Prasse (1957a, b, 1958, 1960) under laboratory conditions. Paschalidis (1974) presented laboratory and field data on several species from Africa.

Sisyphus schaefferi is essentially coprophagous, although other foods can enter into its diet, such as 
mushrooms (Zunino 2017). However, several aspects of its epigean behavior in the wild remain uncertain, especially regarding the role played by the sexes in brood ball construction, rolling, and burying. No definitive information about these topics is available in the literature for S. schaefferi. Our research aimed to investigate these behavioral aspects.

Halffter and Matthews (1966) reported a relationship between the diameter of the dung ball and body length in different roller scarabaeine genera, while Paschalidis (1974) studied the same relationship in seven southern African Sisyphus species. In view of the role of the metatibiae in fashioning the balls (Halffter and Matthews 1966; Halffter in Morón 2003), we studied the ratios of body length to metatibial length, body length to ball weight, and body weight to ball weight in $S$. schaefferi. The purpose was to determine if ball size is isometric in relation to the metatibial and body development ( $c f$. Prange 1977).

The studies by Prasse (1957a, b, 1958, 1960) were performed under laboratory conditions, and the beetles acted, supposedly, in a low population density environment. In contrast, we studied the beetle's behavior in the field in a relatively higherdensity environment (50-70 individuals per pad). We hypothesize that, in this situation, a pair could be forced to deal with multiple attacks during a single ball rolling process.

Our field study sought to investigate the possibly different roles of males and females in rolling behavior. Finally, we performed a statistical analysis of the relationships between the physical parameters of the animals and their balls (weight and volume) and of some traits of their epigean behavior.

\section{Material and Methods}

The study area is located within La Mandria Regional Park, extending $c a .3,100$ ha at the western border of the Po Valley $10 \mathrm{~km}$ north of Turin $\left(45^{\circ} 10^{\prime} \mathrm{N}, 7^{\circ} 75^{\prime} \mathrm{E}\right)$ in northwestern Italy. It lies on a high plain (254-402 m above sea level) and is a mosaic of wooded and open patches with approximately equal extension. Woods are essentially dominated by a Quercus-Carpinetum community, while open habitats are characterized by heather and grassy vegetation.

The large mammalian fauna of La Mandria Regional Park consists of red deer [Cervus elaphus Linnaeus, 1758; Artiodactyla: Cervidae], fallow deer [Dama dama (Linnaeus, 1758); Cervidae], wild boar [Sus scrofa Linnaeus, 1758; Artiodactyla: Suidae], badger [Meles meles (Linnaeus, 1758); Carnivora: Mustelidae], red fox [Vulpes vulpes (Linnaeus, 1758); Carnivora: Canidae], and hare [Lepus europaeus Pallas, 1778; Lagomorpha:
Leporidae]. A total of 246 red deer were counted in 2016 and 150 (underestimated data) in 2017; 39 fallow deer were counted in 2016 and 40 in 2017. The wild boar population was large, but its real size was not correctly estimated because of technical bias (Focardi et al. 2001); it was estimated by the number of animals selectively killed in 2016 (576) and in 2017 (682). Although the badger population size was unknown, the species is widespread in the Park (Rizzotto et al. 2003); one main lair (permanently occupied) and two secondary lairs were present along the two traveled transects (see below). Recent data on the fox population size are not available, but field observations and a footprint survey indicate that the park supports a large and ubiquitous population of this species (M. Rizzotto, personal observation). Hares are very scarce in the study area (M. Rizzotto, personal observation).

Since $S$. schaefferi has a diurnal pattern of activity, observations were made between 10:00am and 7:00pm once a week. The field work was carried out during two periods. From 2012 to 2015, the epigean behavior of $S$. schaefferi was filmed. The activity of each beetle was recorded by means of high-resolution videos $(1,920 \times 1,080$ pixels $)$ obtained with three reflex cameras: Canon Eos 60D, Eos 70D, and Eos 80D equipped with Canon 100$\mathrm{mm}$ macro lens. An artificial LED light was used only on the Eos $80 \mathrm{D}$. These recorded observations allowed us to study the feeding, rolling, and fighting behaviors and combat length of beetles and led us to organize the subsequent collection of numerical data by means of captures from April to September during 2016 and 2017.

Meteorological data were provided by the Agenzia Regionale per la Protezione Ambientale del Piemonte. During the two survey periods (2016 and 2017), the mean monthly temperature ranged between $15.2{ }^{\circ} \mathrm{C}$ and $23.1{ }^{\circ} \mathrm{C}$, the maximum daily temperature between $28.9{ }^{\circ} \mathrm{C}$ and $35.2^{\circ} \mathrm{C}$, the minimum daily temperature between $0.7{ }^{\circ} \mathrm{C}$ and $10.9{ }^{\circ} \mathrm{C}$, and the mean monthly rainfall between $0.3 \mathrm{~mm}$ and $10.8 \mathrm{~mm}$.

From 2012 to 2017, two transects of 3,295 $\mathrm{m}$ and $551 \mathrm{~m}$ were set out in wooded patches commonly used as shelter areas by red deer, fallow deer, and wild boar, mainly females with young. Observations were carried out by walking along the transect while looking for feces and beetles within a band of 30-40 m; beetles were identified by eye.

Temperatures were recorded by means of a TGFA 30.5007 thermo-hygrometer (declared resolution $0.1{ }^{\circ} \mathrm{C}$ and $1 \%$ relative humidity) placed on dry ground and in the shade near the observed individuals. Wind speed was estimated by means of the Beaufort scale (www.arpae.it).

Sex identification, body length (during 2016 and 2017), and metatibial length (during 2016 only) 
were determined by using a $10 \times$ magnifying glass provided with an internal LED light and $0.1 \mathrm{~mm}$ graduated grid (grid length $3 \mathrm{~cm}$ ). To determine sex, the differences in shape and size of the metafemoral tubercle between males and females (Haaf 1955) are useful characters. Tubercle wear was used to ascertain whether the examined individuals had recently emerged or belonged to the previous generation. Body length was measured as the distance from the anterior edge of the pronotum to the dorsal margin of the pygidium. We excluded the head because living individuals often moved it up and down during the measurements. The recorded value was the mean of three measurements of the same individual. To obtain additional measurements of metatibial length, we gathered data from 30 males collected in the same area during 2015, i.e., not as part of the present study.

Individual body weight and ball weight were measured with a Keen Optics KOP 24384 electronic scale (resolution scale $0.01 \mathrm{~g}$ ). Individual specimens were placed in a small, plastic, cylindrical, sealed box, and the box with the specimen was weighed; the animal's weight was taken as the difference between gross weight and tare. All measurements were taken in the shade and sheltered from the wind.

To measure ball dimensions, we assumed that they were similar to an ellipsoid. Thus, three diameters were measured with a Wiha DigiMax 411.1701 digital caliper (reading $0.01 \mathrm{~mm}$ ). Maximum ball diameter was obtained, and the volume was calculated by approximation using the ball diameter in the standard formula.

Hereafter, we use the terms "ball" for the spherical dung mass fashioned by the individuals and "pellet" for the small dung mass utilized without any manipulation. Following Halffter and Matthews (1966), food balls were made for alimentary purposes and brood balls for reproduction. For each measured ball/pellet, the mammalian species that produced the dung was recorded. For each observed beetle pair, we recorded the sex of the two different roles: the beetle positioned in front of the ball and moving backward was defined as the pulling individual and the one positioned behind the ball and moving forward as the pushing individual.

The single individuals or pairs were caught sufficiently far from the dung pad at the end of the ball rolling process after any possible fighting with other individuals. This allowed us to be sure to catch the winner of each competition. To obtain a better statistical comparison, we also measured the body length and body weight of a number of single individuals found along the transect but not engaged in ball construction or transportation.

For measurement of the beetles, the animals were gripped at the metasternal level by using soft entomological forceps. All captured individuals were released after measurements, so no individual was killed.

Generalized linear models (GLM) were used to test for differences between parameters of the dependent variable (body weight) as a function of a categorial factor (male versus female in population; single male versus paired male; single female versus paired female) and a continuous variable (body length). We used GLM analyses to test for differences between metatibial lengths as a function of a categorial factor (sex) and continuous variables (body weight and length). Furthermore, GLM was also used to test for differences in maximum diameter, volume, and weight between brood balls and food balls. Finally, GLM analysis related the balls' maximum diameter, volume, and weight with temperature and humidity. To describe dependent variables, the best distribution was assumed to be Gamma. Parameters were related to explanatory variables via a logarithmic link function (Zuur et al. 2009). The analyses were performed with R software ( $\mathrm{R}$ Core Team 2010). To determine the linear dependence between two variables, we adopted the Pearson correlation $(r)$, which assumes normal data distribution.

\section{Results}

In total, 413 S. schaefferi individuals (272 males and 141 females) were caught during the two years (2016 and 2017): 77 pairs with a brood ball, one pair whose ball was lost, and 132 single individuals with a food ball (105 males and 27 females). During 2017, 125 single individuals (89 males and 36 females) lacking a ball were measured.

The earliest individuals appeared on 25 April 2016 and the last capture occurred on 10 September 2017. Wear of the metafemoral tubercle allowed us to determine if an individual belonged to the "past" or "newly emerged" generation. In 2017, the first newly emerged individual was found on 4 June 2017, while the last old individual was recorded on 6 August 2017.

The species is active across a wide range of temperatures and relative humidities (Martín-Piera and López-Colón 2000). During the periods we observed $S$. schaefferi, temperature varied between $15.8{ }^{\circ} \mathrm{C}$ and $31.3{ }^{\circ} \mathrm{C}$, and relative humidity ranged from $17 \%$ to $68 \%$. Individuals were observed flying in wind velocities between $0 \mathrm{~m} / \mathrm{sec}$ and a light breeze on the Beaufort scale $(3.3 \mathrm{~m} / \mathrm{sec})$.

In the field, individuals making brood balls were observed from 25 April 2016 to 24 July 2016 and from 14 May 2017 to 23 July 2017. During these two periods, individuals making food balls were also observed. After these two dates in July, only food balls were recorded.

The minimum body weight was $0.01 \mathrm{~g}$, while the maximum was $0.28 \mathrm{~g}($ mean $=0.13 \mathrm{~g}, n=413)$. The 
Table 1. Relationships of metatibial length (TL) and live weight (LW) to body length (BL) of adult Sisyphus schaefferi observed in La Mandria Regional Park, Italy, as modeled by the formula $y=a x^{b}$.

\begin{tabular}{lccccc}
\hline \multicolumn{1}{c}{$\boldsymbol{n}$} & $\boldsymbol{y}$ & $\boldsymbol{a}$ & $\boldsymbol{x}$ & $\boldsymbol{b}$ & $\boldsymbol{R}^{2}$ \\
\hline $123(*)$ & TL & 0.4886 & BL & 1.0322 & 0.6876 \\
$315(* *)$ & LW & 0.0003 & BL & 2.7644 & 0.6246 \\
$154(* *)$ & LW & 0.0003 & BL & 2.7981 & 0.5712 \\
\hline
\end{tabular}

* year 2016 mating and non-mating individuals (78 males and 45 females).

** year 2017 mating and non-mating individuals (220 males and 95 females).

*** years 2016-2017 mating pairs only (77 males and 77 females).

minimum body length was $6.2 \mathrm{~mm}$ and the maximum was $12.7 \mathrm{~mm}($ mean $=9.1 \mathrm{~mm}, n=413)$.

The relationships between metatibial length, body weight, and body length are shown in Table 1 and Fig. 1. The GLM analysis showed that the variable best predicting variation in body length was metatibial length $(p<0.001)$ and not the sex of the animal (Table 2). Body weight and body length were not significantly different between the sexes. Nevertheless, body weight increased significantly $(p<0.001)$ in relation to body length (Table 2$)$. However, males and females in pairs had greater weight and length $(p<0.001)$ than specimens not part of a reproductive pair (Table 2).

From the many observed fights, we studied 16 recorded sequences (each comprising many single combats) in which the fighters were automarked (missing legs or semi-permanent dung spots on the body) and thus easily recognizable. When the combat started, the attacked individual reacted by kicking with the hind legs against the striker; afterwards, it tried to climb on the back of the opponent, holding it with the legs. At this point, the attacker could move the defender away from the ball, or, alternatively, both fighters could fall down together. The combat could proceed with prolonged "boxing" (see also Prasse 1958).

We observed that combat over brood balls most frequently involved two opponents. However, under some circumstances, three to six individuals could be involved at the same time. In one case, a male was beaten and replaced by the attacking

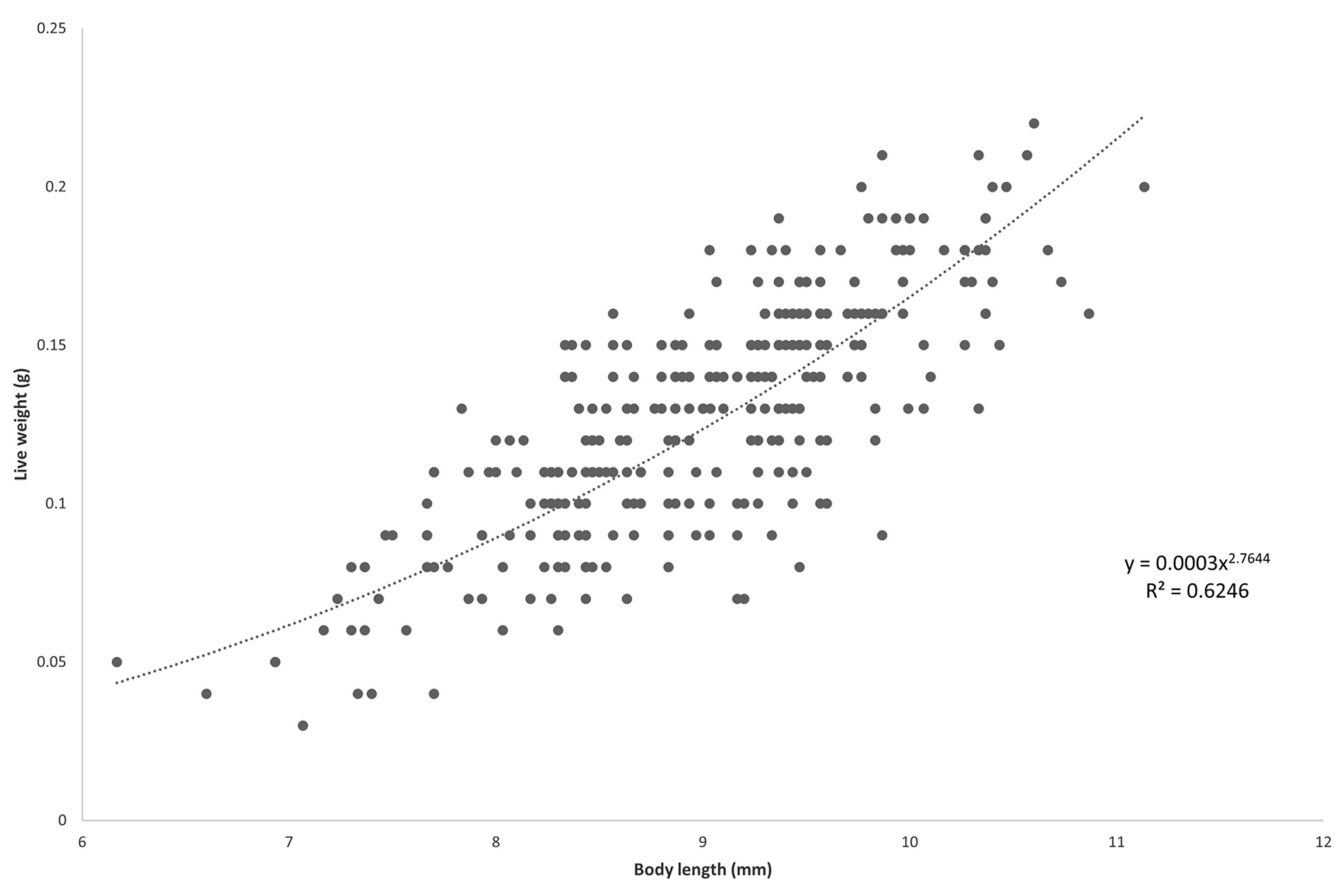

Fig. 1. Scatter plot for live body weight as a function of body length of mating and non-mating adult Sisyphus schaefferi $(n=315)$ observed in La Mandria Regional Park, Italy, during 2017. 
Table 2. Change coefficients of body length, standard error (SE), and statistical significance (GLM) as a function of live weight and metatibial length of adult Sisyphus schaefferi observed in La Mandria Regional Park, Italy. In this parameter estimation analysis, females (in population) and males in couple or females in couple (in single sex analysis) were used as reference categories.

\section{Dependent variable: live weight \\ Distribution: gamma \\ Link-function: LOG}

\begin{tabular}{|c|c|c|c|}
\hline & Coefficient & SE & $\boldsymbol{P}$ \\
\hline \multicolumn{4}{|l|}{ Population* } \\
\hline Male vs. female & -0.04 & 0.02 & 0.14 \\
\hline Body length & 0.28 & 0.01 & $<0.001$ \\
\hline \multicolumn{4}{|l|}{ Male** } \\
\hline Single male vs. male in couple & -0.10 & 0.03 & $<0.001$ \\
\hline Body length & 0.29 & 0.02 & $<0.001$ \\
\hline \multicolumn{4}{|l|}{ Female $* * *$} \\
\hline Female vs. female in couple & -0.14 & 0.04 & $<0.001$ \\
\hline Body length & 0.29 & 0.02 & $<0.001$ \\
\hline
\end{tabular}

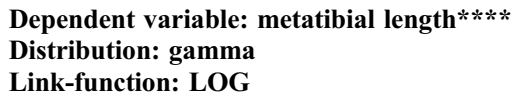

\begin{tabular}{lccc}
\hline & Coefficient & SE & $\boldsymbol{P}$ \\
\hline Population & & & 0.94 \\
Male vs. female & -0.01 & 0.01 & 0.94 \\
Body length & 0.11 & 0.01 & $<\mathbf{0 0 0 1}$
\end{tabular}

* years 2016-2017 mating and non-mating individuals (252 males, 129 females).

** years 2016-2017 paired males 78, single males 174 .

*** years 2016-2017 paired females 78, single females 51 .

**** year 2016 mating and non-mating individuals (78 males, 45 females).

beetle. In nine instances, rolling beetles (both singles rolling a food ball and pairs rolling a brood ball) were attacked by another individual attempting to steal the ball. When the fighting occurred between an attacking individual and a pair, both the pushing and pulling individuals could be involved, in many cases in succession or at the same time.

Most single fights (one-on-one combats) lasted 2-83 seconds $(n=47)$, although there were four other exceptionally long fights $(115-1,163 \mathrm{sec}-$ onds). Overall, $35.3 \%$ of the fights lasted $0-10$ seconds. A single reproductive pair could face a very long period of successive combats: the longest recorded period involved a series of single fights for a total span of 32 minutes.

We obtained the differences in body weight and body length between the partners of 78 reproductive pairs. Body weight differences varied by $0.00-0.13 \mathrm{~g}$ (mean $=0.036 \mathrm{~g}$ ) and body length differences by $0.00-3.03 \mathrm{~mm}($ mean $=0.86 \mathrm{~mm})$. The frequency distributions show mainly low or no differences for both body weight and body length (Table 3).

During the rolling phase, males were pushing in $53.9 \%$ of cases $(n=42)$. However, sometimes the pushing and pulling roles were reversed when the pair encountered a strong obstacle or faced a disturbance. Overall, $57.7 \%$ of pushing individuals ( $n=45$, irrespective of sex) weighed more than pulling individuals. However, only $35.7 \%(n=15)$ of the pushing males weighed more than their partner, while $55.6 \%$ of the pushing females $(n=20)$ weighed more than their partner. The greatest difference observed between the weight of the individual in the puller role and the individual in the pusher role was $0.13 \mathrm{~g}$, with a mean difference of $0.010 \pm 0.045$ g. Overall, $38.5 \%$ of the pushing individuals $(n=30$, irrespective of sex $)$ were longer than the pulling ones. However, only $31.0 \%$ of the pushing males $(n=13)$ were longer than their partner, while $52.8 \%$ of the pushing females $(n=19)$ were longer than their partner. The difference 
Table 3. Differences in length and weight of mating partners $(n=78)$ of Sisyphus schaefferi observed in La Mandria Regional Park, Italy during 2016-2017.

\begin{tabular}{|c|c|c|c|c|c|}
\hline \multicolumn{3}{|c|}{ Length differences (mm) } & \multicolumn{3}{|c|}{ Weight difference (g) } \\
\hline Class & Frequency & $\%$ & Class & Frequency & $\%$ \\
\hline $0.0-0.5$ & 28 & 35.9 & $0.00-0.02$ & 32 & 41.0 \\
\hline $0.5-1.0$ & 20 & 25.6 & $0.02-0.04$ & 16 & 20.5 \\
\hline $1.0-1.5$ & 19 & 24.4 & $0.04-0.06$ & 16 & 20.5 \\
\hline $1.5-2.0$ & 7 & 9.0 & $0.06-0.08$ & 8 & 10.3 \\
\hline $2.0-2.5$ & 3 & 3.9 & $0.08-0.10$ & 2 & 2.6 \\
\hline $2.5-3.1$ & 1 & 1.3 & $0.10-0.12$ & 2 & 2.6 \\
\hline & & & $0.12-0.14$ & 2 & 2.6 \\
\hline Total & 78 & 100 & & 78 & 100 \\
\hline
\end{tabular}

observed between the length of the individual in the puller role and the individual in the pusher role varied from $+1.67 \mathrm{~mm}$ to $-3.03 \mathrm{~mm}$, with a mean difference of $0.265 \pm 1.032 \mathrm{~mm}$. GLM analysis revealed no significant differences in body weight and length between males and females.

We measured 209 molded balls and pellets (187 molded balls, $89.4 \%$; 22 pellets, $10.6 \%$ ), consisting of 77 brood balls ( 70 molded balls, $90.9 \%$; 7 pellets, $9.1 \%$ ) and 132 food balls (117 molded balls, 88.6\%; 15 pellets, $11.4 \%$ ). Among these 209 balls/pellets, $26.0 \%$ were made from badger feces, $62.0 \%$ from wild boar feces, and $12.0 \%$ from red deer feces. The proportion of brood balls made from each dung type seemed to be the same during 2016 and 2017. As only red deer and wild boar release pellets, $S$. schaefferi individuals could only find pellets of these two species. As previously reported by Rizzotto and Barbero (2016), fallow deer feces were not utilized. Hares are very scarce in the park, and we never encountered hare pellets along the transects. We observed an interesting case in which some individuals fed on and tried to make a ball with a dead snail.

Field observations showed that, in nature, individuals fed directly on the dung pad, and this behavior occurred when the dung was excessively rich in large undigested residues (seed and fruit coats, beetle elytra). Undigested graminoid fibers did not prevent ball construction, but in many cases forced the beetles to make asymmetrical balls.

Means and standard deviations of molded ball volume, maximum diameter, weight, and the ratios of brood ball weight/male body weight, brood ball weight/female body weight, and food ball weight/ body weight are reported in Table 4 . The relationships of brood ball and food ball weight versus body weight/body length seem to be clearly isometric in males but less isometric in females (Table 5). Brood balls were larger than food balls, with a significantly higher volume, maximum diameter, and weight (Table 6).

GLM analysis related the maximum diameter, volume, and weight of the brood balls $(n=70)$ to temperature and humidity. There was a significant effect of temperature on brood ball size with maximum diameter, volume, and weight decreasing as temperature increased (Table 7). Relative humidity had no significant effect on brood ball size.

A similar analysis was carried out for food balls $(n=113)$ (Table 8). The model is significant for all the variables in relation to relative humidity but not temperature. When the air humidity rises, food ball volume, weight, and maximum diameter increase. A more detailed analysis showed that during the reproductive season (25 April to 24 July)

Table 4. Characteristics of molded balls (years 2016-2017; brood balls, $n=70$; food balls, $n=117$ ) created by Sisyphus schaefferi observed in La Mandria Regional Park, Italy. Ball weight in grams; volume in $\mathrm{mm}^{3}$; diameter = ball maximum diameter $(\mathrm{mm}) . \mathrm{B} / \mathrm{MW}=$ brood ball weight $/ \mathrm{male}$ live weight ratio; $\mathrm{B} / \mathrm{FW}=$ brood ball weight/female live weight ratio; $\mathrm{B} / \mathrm{W}=$ food ball weight/live weight ratio.

\begin{tabular}{|c|c|c|c|c|c|c|c|c|c|}
\hline & \multicolumn{5}{|c|}{ Brood balls } & \multicolumn{4}{|c|}{ Food balls } \\
\hline & Weight & Volume & Diameter & B/MW & B/FW & Weight & Volume & Diameter & $\mathbf{B} / \mathbf{W}$ \\
\hline Mean & 1.0930 & $1,187.3165$ & 14.1500 & 7.1537 & 7.6554 & 0.7691 & 754.5985 & 12.3456 & 6.9348 \\
\hline SD & \pm 0.5574 & \pm 512.4890 & \pm 2.0583 & \pm 3.3110 & \pm 4.3036 & \pm 0.4081 & \pm 352.5093 & \pm 2.1271 & \pm 3.6586 \\
\hline
\end{tabular}


Table 5. Relationships of rolled brood ball (BB, $n=70)$ and food ball (FB, $n=117)$ weight to live body weight and body length of adult Sisyphus schaefferi observed in La Mandria Regional Park, Italy, as modeled by the formula $y=$ $a x^{b} . \mathrm{LV}=$ live weight; $\mathrm{BL}=$ body length; $r=$ Pearson test value.

\begin{tabular}{rrrlcccr}
\hline $\boldsymbol{n}$ & $\boldsymbol{y}$ & $\boldsymbol{a}$ & \multicolumn{1}{c}{$\boldsymbol{x}$} & $\boldsymbol{b}$ & \multicolumn{1}{c}{$\boldsymbol{R}^{\mathbf{2}}$} & $\boldsymbol{r}$ & $\boldsymbol{p}$ \\
\hline 77 & BB & 9.4639 & male LV & 1.2066 & 0.2309 & 0.49 & $<\mathbf{0 . 0 0 1}$ \\
77 & BB & 0.0008 & male BL & 3.1443 & 0.1907 & 0.41 & $<\mathbf{0 . 0 0 1}$ \\
77 & BB & 3.841 & female LV & 0.7202 & 0.1189 & 0.45 & $<\mathbf{0 . 0 0 1}$ \\
77 & BB & 0.0134 & female BL & 1.9238 & 0.0966 & 0.35 & $<\mathbf{0 . 0 1}$ \\
132 & FB & 3.3074 & LV & 0.7319 & 0.22 & & \\
132 & FB & 0.0019 & BL & 2.6674 & 0.1912 & & \\
\hline
\end{tabular}

the relationships between food ball size and weight and relative humidity were not significant, whereas the relationships were highly significant during the non-reproductive season (6 August to 10 September).

If the pair was not established during brood ball construction, the builder rolled it $50-100 \mathrm{~cm}$ and then stood motionless on the ball, waiting for a partner. In some cases $(n=6)$, the builder waited for the partner for 12-50 minutes and then rolled the ball away and buried it alone. During 2017, this behavior was observed from 2 June to 10 September.

\section{Discussion}

Based on our field observations, the reproductive season for $S$. schaefferi appears to begin in late April/early May and end in the second half of July. This seasonality agrees with what Halffter and Matthews (1966) discussed. No nesting pairs were observed after this period, so we suppose that August and the first half of September are spent by adults in feeding and by larvae in developing to the adult stage. During September, newly emerged adults begin to feed and prepare to overwinter by undergoing diapause.
In our study area, $S$. schaefferi feeds mainly on wild boar, badger, and red deer feces. Moreover, as reported for other areas in northeastern Italy (Melloni 2003), individuals in the study area in previous years were also observed feeding on red fox and horse feces, while fallow deer feces were not utilized (Rizzotto and Barbero 2016). Prasse (1957a) found that, under laboratory conditions, pig feces were not suitable for $S$. schaefferi, although our research showed that wild boar feces are the most utilized food source. This seems to depend, in part, on the density of the different mammalian species, but also on the different size of the scat. Beetles seem to prefer the larger mass of wild boar feces, and they favor male red deer pellets rather than the smaller pellets made by females (Rizzotto and Barbero 2016).

The analysis of body length and weight in males and females showed that these characters are not sexually dimorphic, confirming that sexual dimorphism is manifested only in the shape and size differences of the tubercle on the male and female metafemora (Haaf 1955). Males and females are equally involved in competitive fights during feeding and reproductive periods (Prasse 1958; our field observations). Consequently, the absence of any sexual dimorphism in body length and weight is

Table 6. Comparison of brood balls $(n=70)$ and food balls $(n=117)$ made by Sisyphus schaefferi observed in La Mandria Regional Park, Italy during 2016-2017: change coefficients, standard error (SE), and statistical significance (GLM) for maximum diameter, volume, and weight. In this parameter estimation analysis, food ball was used as reference category.

\begin{tabular}{|c|c|c|c|}
\hline \multicolumn{4}{|l|}{ Distribution: gamma } \\
\hline Link-function: LOG & Coefficient & $\mathbf{S E}$ & $\boldsymbol{P}$ \\
\hline \multicolumn{4}{|l|}{ Maximum diameter } \\
\hline Brood ball vs. food ball & 0.14 & 0.02 & $<0.001$ \\
\hline \multicolumn{4}{|l|}{ Volume } \\
\hline Brood ball vs. food ball & 0.45 & 0.07 & $<0.001$ \\
\hline \multicolumn{4}{|l|}{ Weight } \\
\hline Brood ball vs. food ball & 0.35 & 0.08 & $<0.001$ \\
\hline
\end{tabular}


Table 7. Rolled brood balls $(n=70)$ observed in La Mandria Regional Park, Italy during 2016-2017: change coefficients, standard error (SE), and statistical significance (GLM) for maximum diameter, volume, and weight as a function of temperature and humidity.

\begin{tabular}{lccc}
\hline Distribution: gamma & & SE & $\boldsymbol{P}$ \\
\hline Link-function: LOG & Coefficient & 0.01 & $<\mathbf{0 . 0 0 5}$ \\
\hline Maximum diameter & & 0.66 \\
Temperature & -0.01 & 0.20 & $<\mathbf{0 0 1}$ \\
Humidity & -0.09 & 0.02 & 0.81 \\
Volume & & 0.57 & $<\mathbf{0 . 0 0 1}$ \\
Temperature & -0.06 & & 0.64 \\
Humidity & -0.14 & 0.02 & 0.61 \\
Weight & & & \\
Temperature & -0.09 & & \\
Humidity & 0.29 & & \\
\end{tabular}

probably related to the lack of differences in the roles of males and females in combat.

Dung balls are made for both feeding and breeding purposes. Nevertheless, food balls can be built several times by one individual during its adult life, whereas only one brood ball is available to the individual during its larval life. Thus, it is possible that food and brood balls are made by different tactics. In contrast to Halffter and Matthews (1966), but according to what was reported by Halffter et al. (2011) for some species of Scarabaeus Linnaeus, the comparison between weight and volume of brood and food balls formed by $S$. schaefferi clearly shows that the former are significantly larger and heavier than the latter. This should indicate that individuals dedicate more effort to brood ball construction than to making food balls. Food balls require less care because they may be made under a wider range of conditions (temperature, dung characteristics, competition) than brood balls.

During the non-reproductive season, food ball volume and weight increase as relative humidity

Table 8. Rolled food balls observed in La Mandria Regional Park, Italy during 2016-2017: change coefficients, standard error (SE), and statistical significance (GLM) for maximum diameter, volume, and weight as a function of temperature and humidity.

\begin{tabular}{|c|c|c|c|c|c|c|c|c|c|}
\hline \multirow{2}{*}{$\frac{\text { Distribution: gamma }}{\text { Link-function: LOG }}$} & \multicolumn{3}{|c|}{ Total sample } & \multicolumn{3}{|c|}{ During breeding } & \multicolumn{3}{|c|}{ Non-breeding } \\
\hline & Coefficient & SE & $\boldsymbol{P}$ & Coefficient & SE & $P$ & Coefficient & SE & $P$ \\
\hline \multicolumn{10}{|l|}{ Maximum diameter } \\
\hline Temperature & -0.01 & 0.01 & 0.83 & 0.01 & 0.01 & 0.39 & -0.01 & 0.01 & 0.44 \\
\hline Humidity & 0.53 & 0.15 & $<0.001$ & 0.15 & 0.26 & 0.56 & 0.76 & 0.22 & $<0.01$ \\
\hline \multicolumn{10}{|l|}{ Volume } \\
\hline Temperature & 0.01 & 0.02 & 0.65 & 0.01 & 0.02 & 0.81 & -0.01 & 0.01 & 0.85 \\
\hline Humidity & 1.82 & 0.57 & $<0.001$ & 0.65 & 0.70 & 0.36 & 2.19 & 0.61 & $<0.001$ \\
\hline \multicolumn{10}{|l|}{ Weight } \\
\hline Temperature & -0.02 & 0.02 & 0.17 & -0.02 & 0.02 & 0.37 & -0.03 & 0.02 & 0.19 \\
\hline Humidity & 2.35 & 0.46 & $<0.001$ & 0.97 & 0.72 & 0.19 & 2.87 & 0.71 & $<0.001$ \\
\hline
\end{tabular}

Total sample, $n=113$ from 25 April to 10 September.

During breeding, $n=35$ from 25 April to 24 July.

Non-breeding, $n=78$ from 6 August to 10 September. 
rises, presumably because the beetle can incorporate more water in the ball. In contrast, brood ball volume and weight decrease when temperature increases. We conjecture that the beetle tends to utilize drier dung in order to furnish the larva with more nutrient-rich food. During the reproductive season, food ball size and weight are not influenced by air temperature and relative humidity.

Since individuals can feed directly on the dung pad (as stressed by Prasse 1957a), food ball construction becomes optional. Filmed field observations also suggest that more abundant, moist, and soft feces facilitate direct feeding, whereas intraspecific competition increases when feces are dry and hard; thus, food ball construction becomes more frequent in this instance.

Since the legs (mainly the hind legs) play a pivotal role during ball construction (Halffter and Matthews 1966; Morón 2003), we investigated the relationship between metatibial length and adult body length. Interestingly, increased metatibial length is isometric in relation to increased body length. The equations describing the relationships of increased brood ball weight with male body size and weight have an exponent, showing that the relation is isometric. This seems to be clearly related to the isometric relationship between metatibial length and body length. The equations describing the relationships of increased brood ball weight with male body length and weight are different from the equations for females, in terms of both data dispersion and the lower equation exponent. Nevertheless, the Pearson test showed that females make significantly heavier and larger brood balls rather than heavier and larger males.

As mentioned above, the role of the two sexes in making brood balls is still uncertain. In the field, it is difficult to clearly determine the sex of the ball builder without interrupting the building process; moreover, it is impossible to establish if the ball is a food ball or brood ball before the eventual forming of the pair. Field observations show that one individual starts the ball construction, and then the second individual of the opposite sex joins in. Courtship was never observed, but the beetles recognize one another very rapidly ( 0.5 second $)$ (Prasse 1957b). The second individual could collaborate or not in ball construction; in any case, the time it spends does not span the entire ball construction period. The builder's strategy seems to be its primary role in ball making. The other partner has a wider range of choices: it can partially contribute to the construction, or accept the ball without helping the first partner, or not accept the ball at all. Consequently, the size parameters of the ball are more closely related to those of the builder, and the correlation between the size parameters of the ball and those of the second partner are weaker and more variable. On the assumption that either sex can initiate brood ball construction and then await the arrival of a partner, the exponents of the equations relating brood ball size to the length and weight of the individuals should be very similar and isometric. The difference between the equations describing the relationships of brood ball weight versus male (or female) length and weight leads us to hypothesize that males are responsible for ball construction.

In general, ball weight increases directly as male weight and size increase. In view of the role of the metatibiae in the ball construction process, the relationship between ball size and metatibial length becomes clear. The rather wide dispersion of the values can partly be explained as an effect of direct competition (the measured males were not always the ball maker but sometimes the victorious attacker), variation of the physical characteristics of the dung (which can change in relation to climatic conditions, the mammal's diet, and season), and the possible contribution of the female to ball construction.

Studies have investigated the relative contribution of the male and female in the process of brood ball relocation and nesting in scarabaeine roller dung beetles. These works, dealing with Deltochilini (Canthon Hoffmannsegg; see Guertin 1991 and Matthews 1963) and Scarabaeini (Scarabaeus; see Halffter et al. 2011), highlighted the great variability of these behaviors. In some species, the male plays the leading role in brood ball making, and it is exclusively he who rolls, defends, and buries the ball. In other species, the role of the female is somewhat passive, and she collaborates to a varying degree in the building and rolling activities. In $S$. schaefferi, the members of the pair actively collaborate and play two different roles in moving the brood ball: the first individual pushes the ball and the second one pulls it. Prasse (1957b) stated that, under laboratory conditions, $80 \%$ of the pushing individuals were females. However, our field study showed that the sex ratio of pulling and pushing individuals is near 1:1. Weight and body length seem to have no relation to the role played by the individuals. Hence, the two roles can sometimes be reversed during the rolling activity.

Adult size closely depends on the larval development, whereas weight can depend on the physiological and nutritional status of the individual. Weight is related to reproductive status. The Pearson test shows that females do not select longer and heavier males since the correlation coefficients are near nil and only the coefficient relative to body weight is at a low level of significance. Moreover, the relatively wide range of body weight and length between the members of the pairs and the 
level of competitive interactions (varying according to local population density and food availability) explain the fact that smaller individuals are also able to mate.

Paired males and females are longer and heavier than non-paired males and females. Size and weight seem to be determinant factors in fights, as demonstrated for Canthon cyanellus LeConte by Chamorro-Florescano et al. (2011).

Prasse (1958) reported that combat involves only two opponents. Our observations showed that this may be the rule. However, in some circumstances (e.g., high population density or limited food resources), three or more (maximum six) individuals can be involved at the same time. Indeed, artificial laboratory conditions cannot adequately reproduce all the possible natural constraints.

In the literature, it is generally acknowledged that scarabaeine dung beetles are characterized by a high level of interspecific differentiation in terms of both ecology (Halffter and Matthews 1966; Hanski and Cambefort 1991) and behavior (Barbero and Palestrini 2002; Halffter and Edmonds 1982). In contrast, less attention has been given to intraspecific variability, i.e., the ability of single individuals to vary their behavior according to sporadic environmental changes. Our study points out some elements that support strong behavioral plasticity in S. schaefferi. This seems to be true for food choice (coprophagy vs. mycophagy or necrophagy), feeding behavior (directly on the dung pad versus food relocation, conversion of brood balls into food balls), brood ball building (male involved versus female), and brood ball rolling (reversal of pushing/pulling roles). We think that this topic is worthy of future research that focuses on $S$. schaefferi and other dung beetle species.

\section{ACKNowhedgMents}

We are grateful to La Mandria Regional Park for permission to collect beetles. We are indebted to Paolo Debernardi for providing us useful information and Irene Piccini and Beatrice Nervo for field and laboratory assistance.

\section{References Cited}

Barbero, E., and C. Palestrini. 2002. Remarks on subsociality in the Scarabaeinae (Coleoptera: Scarabaeidae). Insect Social Life 4: 205-213.

Chamorro-Florescano, I. A., M. E. Fávila, and R. Macías-Ordoñez. 2011. Ownership, size and reproductive status affect the outcome of food ball contests in a dung roller beetle: When do enemies share? Evolution and Ecology 25: 277-289.
Focardi, S., A. M. De Marinis, M. Rizzotto, and A. Pucci. 2001. Comparative evaluation of thermal infrared imaging and spotlighting to survey wildlife. Wildlife Society Bulletin 29(1): 133-139.

Guertin, D. S. 1991. Atypical sex-role behavior in the ball-rolling dung beetle Canthon pilularius L. (Coleoptera, Scarabaeidae). The Pan-Pacific Entomologist 67(2): 145-146.

Haaf, E. 1955. Über die Gattung Sisyphus Latr. (Col. Scarab.). Entomologische Arbeiten der Museum G. Frey 6: 341-381.

Halffter, G., and W. D. Edmonds. 1982. The Nesting Behavior of Dung Beetles (Scarabaeinae): An Ecological and Evolutive Approach. Instituto de Ecología, México, DF, Mexico, 176 pp.

Halffter, G., V. Halffter, and M. E. Fávila. 2011. Food relocation and the nesting behavior in Scarabaeus and Kheper (Coleoptera: Scarabaeinae). Acta Zoológica Mexicana 27(2): 305-324.

Halffter, G., and E. G. Matthews. 1966. The natural history of dung beetles of the subfamily Scarabaeinae (Coleoptera, Scarabaeidae). Folia Entomológica Mexicana 12-14: 1-312.

Hanski, I., and Y. Cambefort. 1991. Dung Beetle Ecology. Princeton University Press, Princeton, NJ, 481 pp.

Martín-Piera, F., and J. I. López-Colón. 2000. Coleoptera, Scarabaeoidea I. Fauna Ibérica, Vol. 14. Museo Nacional de Ciencias Naturales. CSIC, Madrid, Spain, 528 pp.

Matthews, E. G. 1963. Observations on the ball-rolling behavior of Canthon pilularius (L.). (Coleoptera: Scarabaeidae). Psyche 70: 75-93.

Melloni, L. 2003. Ricerche coleotterologiche nel Parco Nazionale delle foreste casentinesi, Monte Falterona e Campigna (Forli-Cesena): Scarabeoidei coprofagi e isteridi sapro-coprofili (Insecta Coleoptera Scarabaeoidea, Histeridae). Quaderno di Studi e Notizie di Storia naturale della Romagna 18: 83-108.

Morón, M. A. 2003. Atlas de los Escarabajos de México. Vol. II. Argania Editio, Barcelona, Spain.

Paschalidis, K. M. 1974. The Genus Sisyphus Latr. (Coleoptera: Scarabaeidae) in Southern Africa. MSc Thesis, Rhodes University, Grahamstown, South Africa, 183 pp.

Philips, T. K., E. Pretorius, and C. H. Scholtz. 2004. A phylogenetic analysis of dung beetles (Scarabaeinae: Scarabaeidae): Unrolling an evolutionary history. Invertebrate Systematics 18: 53-88. DOI: $10.1071 / \mathrm{IS} 03030$.

Prange, H. D. 1977. The scaling and mechanics of arthropod exoskeletons [pp. 169-181]. In: Scale Effects in Animal Locomotion (T. J. Pedley, editor). Academic Press, New York, NY, 545 pp.

Prasse, J. 1957a. Nahrungserwerb koprophager Pillenwälzer (Sisyphus schaefferi L. und Gymnopleurus geoffroyi Fuessl. Col. Scarab.). Wissenschaftliche Zeitschrift der Martin-Luther-Universität Halle Wittenberg, Mathemathisch-Naturwissenschaftliche Rehie 6(3): 439-444.

Prasse, J. 1957b. Das Brutfursorgeverhalten der Pillenwälzer Sisyphus schaefferi L. und Gymnopleurus geoffroyi Fuessl. (Col. Scarab.). Wissenschaftliche 
Zeitschrift der Martin-Luther-Universität Halle Wittenberg, Mathemathisch-Naturwissenschaftliche Rehie 6(4): 569-614.

Prasse, J. 1958. Die Kämpfe der Pillenwälzer Sisyphus schaefferi (L.) und Gymnopleurus geoffroyi Fuessl. (Col. Scarab.). Wissenschaftliche Zeitschrift der Martin-Luther-Universität Halle Wittenberg, Mathemathisch-Naturwissenschaftliche Rehie 7(1): 89-92.

Prasse, J. 1960. Über den Start und Flug des Sisyphus schaefferi L. (Col. Scarabaeidae). Beiträge zur Entomologie 10(1/2): 168-183.

R Development Core Team. 2010. R: A language and environment for statistical computing. $\mathrm{R}$ Foundation for Statistical Computing, Vienna, Austria. www.R-project.org.

Rizzotto, M., and E. Barbero. 2016. Peeling dung pellets: An unrecorded behavior in the roller dung beetle Sisyphus schaefferi Linnaeus (Coleoptera: Scarabaeidae: Sisyphini). The Coleopterists Bulletin 70(4): 819-821. DOI: 10.1649/0010-065X-70.4.819.

Rizzotto, M., A. M. De Marinis, C. Macocco, C. Giacoma, and L. Tontini. 2003. Il tasso nel Parco Regionale "La Mandria". IV Congr. It. Teriologia. Hystrix, Italian Journal of Mammalogy (suppl.): 70-71.
Tarasov, S. I., and D. Dimitrov. 2016. Multigene phylogenetic analysis redefines dung beetles relationships and classification (Coleoptera: Scarabaeidae: Scarabaeinae). BMC Evolutionary Biology 16, 257. DOI: $10.1186 / \mathrm{s} 12862-016-0822-x$.

Tarasov, S. I., and F. Génier. 2015. Innovative Bayesian and parsimony phylogeny of dung beetles (Coleoptera, Scarabaeidae, Scarabaeinae) enhanced by ontology-based partitioning of morphological characters. PLoS ONE 10(3): e0116671. DOI: 10.1371/ journal.pone.0116671.

Zunino, M. 1992. Food relocation behaviour: A multivalent strategy of Coleoptera [pp. 297-314]. In: Advances in Coleopterology (M. Zunino, X. Belles, and M. Blas, editors). AEC, Barcelona, Spain, 323 pp.

Zunino, M. 2017. Sobre el régimen alimenticio de Sisyphus schaefferi (Linnaeus) (Coleoptera: Scarabaeidae: Sisyphini). Dugesiana 24(1): 25-29.

Zuur, A. F., E. N. Ieno, N. Walker, A. A. Saveliev, and G. M. Smith. 2009. Mixed Effects Models and Extensions in Ecology with R. Springer-Verlag, New York, NY, 574 pp.

(Received 12 September 2019; accepted 3 January 2021. Publication date 20 March 2021.) 